\section{Chagas congénito: necesidad de estrategias integrales para su abordaje}

\section{Congenital Chagas: need for comprehensive strategies for its approach}

\section{Estimado Sr. Editor:}

Hemos leído con interés el artículo de Denegri y cols. ${ }^{1}$. En el mismo se pone de manifiesto los importantes logros en la detección precoz de Chagas congénito en la población atendida en dicho hospital. Los test empleados para el cribado en mujeres embarazadas y la detección en los recién nacidos han demostrado la mejor performance en el diagnóstico de laboratorio, y han podido ser aplicados en el contexto de la complejidad de ese hospital. Sin embargo, en sitios más apartados, donde no se dispone de la infraestructura suficiente, son necesarias estrategias que requieren baja complejidad ${ }^{2}$ : en estadio crónico, la utilización simultánea de dos inmunocromatografías de flujo lateral, ofrece resultados comparables al uso simultáneo de HAI + ELISA ${ }^{3}$. Para el diagnóstico de transmisión al recién nacido, los exámenes directos por observación microscópica, como las versiones de la técnica de Strout adaptada para bajo volumen de sangre, brindan resultados sensibles y específicos, cuando son practicados por profesionales con experiencia ${ }^{4}$. Ya sea que se disponga de una mayor o menor complejidad en el laboratorio, es fundamental disponer de profesionales con la formación suficiente para el diagnóstico e interpretación de técnicas serológicas, moleculares o microscópicas.

Por otro lado, advertimos en el mencionado artículo que una importante proporción de mujeres no completaron el tratamiento etiológico específico. Si bien la seroconversión negativa post tratamiento puede demorar varios años o no producirse en pacientes adultos crónicos, se ha observado que disminuyen las posibilidades de transmisión a la descendencia, como es señalado por los autores.

En este sentido, más allá de la complejidad del centro de salud donde se atienda a la población y de la capacidad e infraestructura de diagnóstico, continúa siendo clave el trabajo interdisciplinario en atención primaria de la salud.

La enfermedad de Chagas tiene una compleja trama de condicionamientos sociales, culturales e históricos, donde las personas suelen ser estigmatizadas o discriminadas afectando su calidad de vida.

Las respuestas requieren de un enfoque multidimen- sional, que integre políticas de promoción de la salud y preventivas, soluciones que respondan a las necesidades de las comunidades y servicios de salud centrados en la persona. La estrategia de atención debe incluir intervenciones que aborden los prejuicios y las representaciones de la enfermedad, tanto de los integrantes del equipo de salud $^{5}$ como de la comunidad; así como el establecimiento de alianzas con organizaciones sociales y grupos de pacientes para aumentar la adherencia al tratamiento en caso de necesitarlo.

\section{Diego Mendicino ${ }^{1} y$ \\ María Alejandra Roman-Miyasato ${ }^{2}$}

${ }^{1}$ Centro de Investigaciones sobre Endemias Nacionales, Facultad de Bioquímica y Ciencias Biológicas, Universidad Nacional del Litoral. Argentina.

${ }^{2}$ Cátedra de Educación para la Salud, Escuela Superior de Sanidad, Universidad Nacional del Litoral.

Argentina.

\section{Referencias bibliográficas}

1.- Denegri M, Oyarce A, Larraguibel P, Ramírez I, Rivas E, Arellano G, et al. Cribado y transmisión congénita de la enfermedad de Chagas en población usuaria del Hospital Dr. Félix Bulnes Cerda y Atención Primaria de Salud del Servicio de Salud Metropolitano Occidente de Santiago, Chile. Rev Chilena Infectol 2020; 37 (2): 129-37.

2.- Messenger L A, Gilman R H, Verastegui M, GaldosCardenas G, Sánchez G, Valencia E, et al. Toward improving early diagnosis of congenital Chagas disease in an endemic setting. Clin Infect Dis 2017; 65 (2): 268-75. doi: 10.1093/ cid/cix277.

3.- Mendicino D, Colussi C, Moretti E. Simultaneous use of two rapid diagnostic test for the diagnosis of Chagas' disease. Trop Doct 2019; 49 (1): 23-6. doi: 10.1177/0049475518813792.

4.- Vera-Ku M, Meza-González G, Carlier Y, Truyens C, Gamboa-León R. Comparison of methodologies for detecting Trypanosoma cruzi parasites by microscopic observation of microhematocrit capillary tubes. Rev Soc Bras Med Trop 2019; 52:e20180505. doi: 10.1590/00378682-0505-2018.

5.- Sanmartino M, Amieva C, Medone P. Representaciones sociales sobre la problemática de Chagas en un servicio de salud comunitaria del Gran La Plata, Buenos Aires, Argentina. Glob Health Promot 2018; 25 (3): 102-110. doi: $10.1177 / 1757975916677189$.

Correspondencia a: alersantafe@gmail.com 


\section{Respuesta}

\section{Estimado Sr. Editor:}

Nos dirigimos a usted con el fin de compartir nuestra experiencia con la comunidad científica en respuesta a los comentarios realizados a nuestra publicación (Denegri y cols. (2020) en Rev Chilena Infectol 37 (2):129-37), los que fueron enviados como Carta al Editor por parte de los investigadores Diego Mendicino ${ }^{I}$ y María Alejandra Roman-Miyasato ${ }^{2}{ }^{1}$ Centro de Investigaciones sobre Endemias Nacionales, Facultad de Bioquímica y Ciencias Biológicas, Universidad Nacional del Litoral. Argentina. ${ }^{2}$ Cátedra de Educación para la Salud, Escuela Superior de Sanidad, Universidad Nacional del Litoral. Argentina.

El Plan Nacional de Prevención y Control de la Enfermedad de Chagas se inició en Chile hace más de cuatro décadas con el fin del control vectorial y luego del control transfusional y vigilancia.

En 1999 el país logró la interrupción de la transmisión vectorial, recertificada el año 2016. Desde el 2014 cuenta con la Norma Técnica $N^{\circ} 162$, la cual orienta sobre las actividades y procesos en los distintos niveles de atención, estableciendo las directrices técnicas para la integración de los diferentes equipos con énfasis en los desafíos pendientes, tales como la pesquisa en mujeres embarazadas y la atención de los pacientes. Consecuentemente, se implementó el tamizaje serológico de las gestantes y la detección de los recién nacidos infectados.

El programa se inició en el norte del país, debido a que se describían como regiones de endemia alta; por tanto, eran las que presentaban las mayores tasas de enfermedad de Chagas en población general y gestante ${ }^{1}$.

Nuestro Servicio de Salud Metropolitano Occidente se encuentra en la Región Metropolitana, en el centro del país y, se define como una zona de endemia intermedia con tasas que varían de 1,5 a 3,5 x 100.000 habitantes ${ }^{1}$. En ésta, el cribado de la población gestante se inició el año 2016, período de inicio que comprende nuestro trabajo de investigación ${ }^{3}$, el cual que se extendió por los dos primeros años de operación del programa.

En Chile, los controles del embarazo incluyen el análisis obligatorio de la enfermedad de Chagas. Su cumplimiento requiere de una red multidisciplinaria que integra la atención primaria con los laboratorios clínicos, que se encuentran adscritos a los hospitales, y con el laboratorio de referencia nacional, dependiente del Instituto de Salud Pública (ISP) de Chile. De esta manera, todo binomio madre-hijo tiene acceso a los exámenes de laboratorio necesarios para diagnosticar la enfermedad de Chagas y detectar precozmente la transmisión transplacentaria del parásito, así como acceder al tratamiento farmacológico.

En el caso del diagnóstico del recién nacido, como la norma nacional lo indica, a todo hijo de madre con serología positiva confirmada, nacido en los hospitales de país, se le realiza la detección del genoma de $T$. cruzi en una muestra de sangre mediante técnica de RPC (reacción de polimerasa en cadena). Además, se acompaña con exámenes serológicos ELISA, RIFI y WB (en caso de discordancias). En los establecimientos que cuentan con profesionales entrenados se realizan también exámenes directos.

En nuestro hospital, todo niño dado de alta se retira con la muestra de sangre tomada para su análisis por RPC, así como para su estudio serológico en el ISP. Sin embargo, nuestro hospital no incluye profesionales expertos en exámenes directos, por ejemplo, micro Strout.

En relación con el tratamiento antiparasitario, cabe mencionar que, a nivel país, la cantidad de mujeres en edad fértil que lo completó el año 2017 fue menor al $60 \%{ }^{2}$. Ello, refleja que el acceso, adherencia y término de la terapia es un problema nacional por resolver. Para ello, pensamos que es clave mejorar la coordinación con la atención primaria, fortalecer los equipos de trabajo y brindar apoyo multidisciplinario a las gestantes infectadas.

En Chile, gran parte de la población desconoce la existencia de $T$. cruzi y del tipo de infección persistente que ocasiona en el ser humano, a pesar de que hemos convivido históricamente con la enfermedad ${ }^{4} \mathrm{~A}$ su vez, y a diferencia de Argentina, en nuestro país no existe la estigmatización o discriminación de las personas, ya que su condición de salud es confidencial, según indica la Ley $\mathrm{N}^{\circ} 20854$ de deberes y derechos de los pacientes.

Marisol Denegri C. ${ }^{1}$ y Nancy Maulén L. ${ }^{2}$ ${ }^{1}$ Médico Parasitólogo, encargada de Policlínico de Chagas, Hospital Dr. Félix Bulnes Cerda, Santiago, Chile; Docente Unidad de Parasitología, Departamento de Pediatría y Cirugía Infantil, Facultad de Medicina Occidente, Universidad de Chile. Santiago, Chile.

${ }^{2}$ Bioquímico, Doctor en Ciencias, encargada Programa Chagas, Laboratorio Clínico, Hospital Dr. Félix Bulnes Cerda. Santiago, Chile.

\section{Referencias bibliográficas}

1.- Ministerio de Salud de Chile (MINSAL). Informe Indicadores Programáticos Plan Nacional Enfermedad de Chagas. 2016 [Internet]. Disponible en: https://diprece. minsal.cl/wrdprss_minsal/wp-content/uploads/2017/06/ ENFERMEDAD-DE-CHAGAS-2016_01.pdf

2.- Ministerio de Salud de Chile (MINSAL). Informe Estrategia Integrada de Prevención y Control de la Enfermedad de Chagas. 2017. [Internet]. Disponible en: https://diprece. minsal.cl/wp-content/uploads/2018/10/FOLLETO_ PROGRAMA-CHAGAS.pdf.

3.- Denegri M, Oyarce A, Larraguibel P, Ramírez I, Rivas E,Arellano G, et al. Cribado y transmisión congénita de la enfermedad de Chagas en población usuaria del Hospital Dr. Félix Bulnes Cerda y Atención Primaria de Salud de Servicio de Salud Metropolitano Occidente de Santiago, Chile. Rev Chilena Infectol 2020; 37 (2): 129-37.

4.- Canals M, y cols. ¿Qué dicen los números de la evolución temporal de la enfermedad de Chagas? Rev Chilena Infectol 2017; 34 (2): 120-7. 